\title{
Prediction model of laparoendoscopic single-site surgery in gynecology using machine learning algorithm
}

\author{
Jun Ma, Jiani Yang, Shanshan Cheng, Yue Jin, Nan Zhang, Chao Wang, Yu Wang \\ Department of Obstetrics and Gynecology, Renji Hospital, School of Medicine, Shanghai Jiaotong University, Shanghai, China
}

Videosurgery Miniinv 2021; 16 (3): 587-596

DOI: https://doi.org/10.5114/wiitm.2021.106081

\begin{abstract}
Introduction: Minimally invasive surgery has been widely used in gynecology. The laparoendoscopic single-site surgery (LESS) risk prediction model can provide evidence-based references for preoperative surgical procedure selection. Aim: To determine whether the patients are suitable for LESS and to provide guidance for the clinical operation plan, we aimed to compare the clinical outcomes of LESS and conventional laparoscopic surgery (CLS) in gynecology. We constructed a LESS risk prediction model and predicted surgical conditions for the preoperative evaluation system. Material and methods: A retrospective analysis was carried out among patients undergoing LESS $(n=1019)$ and CLS $(n=1055)$. Various clinical indicators were compared. Multiple machine model algorithms were evaluated. The optimal results were chosen as the model to form the risk prediction model.

Results: The LESS group showed advantages in the postoperative 12/24 h visual analog scale and Vancouver scar score compared with the CLS group $(p<0.05)$. The comparisons in other clinical indicators between the two groups showed that each group had advantages and the difference was statistically significant $(p<0.05)$, including operative time, estimated blood loss, and hospital stay. We evaluated the predictive value for various models using AUC values of $0.77,0.77,0.76$, and 0.67 for XGBoost, random forest, GBDT, and logistic regression, respectively. The decision tree model was shown to be the optimal model.

Conclusions: LESS can reduce postoperative pain, shorten hospital stay and make scars acceptable. The risk prediction model based on a machine learning algorithm has manifested a high degree of accuracy and can satisfy the doctors' demand for individualized preoperative evaluation and surgical safety in LESS.
\end{abstract}

Key words: laparoendoscopic single-site surgery, prediction model, conventional laparoscopic surgery, machine learning, adverse outcomes.

\section{Introduction}

Laparoendoscopic single-site surgery (LESS) has become widely used in the treatment of gynecologic diseases. It features advantages such as using a single, small incision and providing esthetic wound closure. However, the LESS procedure is not risk-free. Surgical treatments are associated with complications, and new technology does not eliminate complications. The overall complication rate for gynecologic laparoscopic surgery is 0.6\% [1], which is secondary to pelvic adhesions, clinical experience of the surgeon, operative technique, and surgical response. An increasing number of surgeons adapted the LESS despite some patients being poor candidates for the procedure. Surgeons must strictly adhere to the proper indications and contraindications of LESS; esthetic wound closure should not be considered as an indication since it may render LESS a "maximally invasive" instead of a "minimally inva-

\section{Address for correspondence}

Yu Wang, Department of Obstetrics and Gynecology, Renji Hospital, School of Medicine, Shanghai Jiaotong University, Shanghai, China, e-mail: renjiwangyu@126.com 
sive" technique. Different surgical specialists must establish clearly defined rules of operation and ef fective methods of evaluation to guide appropriate clinical decisions regarding the appropriateness of LESS.

Surgeons can combine several forms of patient information, such as the clinical diagnosis, treatment, electronic medical records, and medical data, to accurately understand and plan corresponding management for each disease $[2,3]$. Numerous modalities have emerged to process patient information through numerous efficient clinical decision support systems. Among these is artificial intelligence, represented by machine learning, which has been rapidly gaining recognition in basic and clinical medicine. Machine learning is used to generate models from data, and then mass data. For example, the gradient boosting decision tree (GBDT) is a data mining algorithm incorporating a decision tree and was proposed by Friedman in 1999 [4]. The GBDT is applied in prediction fields with satisfactory results $[5,6]$. The extreme gradient boosting (XGBoost) model algorithm has become one of the common tools for solving classification problems owing to its efficiency, flexibility, and accuracy [7]. A random forest is an ensemble learning algorithm of machine learning with the basic unit of a decision tree, which can independently train some relatively weak learning models through ensemble learning, integrate the results, and realize overall prediction [8]. These three models are decision tree models. A logistic regression (LR) algorithm is a linear model which is widely used in different fields as an important classification method supporting class result output and has become one of the most frequently used methods in data mining and data analysis applications because of its fast computation speed and easy understandability [9]. The above-mentioned algorithms have different prediction roles and values in medicine.

We conducted a retrospective study to compare the clinical outcomes of LESS and conventional laparoscopic surgery (CLS) in gynecological diseases. Additionally, we analyzed the usability of data on patients who underwent LESS and CLS generated via machine learning then analyzed the generated algorithms to select the optimal model to predict surgical conditions for the preoperative evaluation system of LESS. Our study is an innovation towards precision diagnosis and treatment in the gynecology department involving the LESS.

\section{Aim}

The learning task of our study is to determine whether the patients are suitable for LESS and to provide guidance for the clinical operation plan by a machine learning model. We aimed to compare the clinical outcomes of LESS and CLS in gynecological diseases. We constructed a LESS risk prediction model to predict surgical conditions for the preoperative evaluation system of LESS.

\section{Material and methods}

\section{Patients}

A total of 1019 patients with various gynecologic diseases who underwent LESS since the procedure was first adopted in 2015 and 1055 patients who underwent CLS in the Department of Obstetrics and Gynecology, Renji Hospital, School of Medicine, Shanghai Jiaotong University, December 2015 to December 2020 were studied retrospectively. The inclusion criteria of subjects for this study were as follows: (1) histologically confirmed gynecological diseases; (2) underwent standardized LESS or CLS surgery. Patients were excluded from our study if (1) their follow-up observation or records were unavailable; (2) incomplete clinical, laboratory, or imaging data records; (3) comorbidities such as hematological disorders or were administered medications that can influence coagulation function within 1 month. We classified patients into four surgical types in both the LESS and CLS groups, including adnexal surgery, myomectomy, hysterectomy, and malignant tumor surgery. Because the study was an observational, non-interventional retrospective analysis that did not affect patient management, it was exempt from institutional review board approval.

\section{Descriptive statistics and data exploration}

Eleven clinical indicators before surgery were screened and incorporated into the analysis: age, body mass index (BMI), height, weight, history of abdominal surgery, surgical method, cyst size (maximum diameter), fibroid size (maximum diameter), pathologic type, preoperative hemoglobin $(\mathrm{Hb})$ concentration, and surgical experience of the surgeon.

Data for surgical outcomes, including operative time, estimated blood loss, changing hemoglobin level $(\delta \mathrm{Hb})$, hospitalization time, $12 / 24 \mathrm{~h}$ visual analog scale (pain scores ranging from 0 to 10 points measured $12 \mathrm{~h}$ and $24 \mathrm{~h}$ after surgery), Vancouver scar score (VSS) 
[10], and adverse outcomes of the LESS/CLS. The operative time was defined as the duration from the first incision to the final closure. Estimated blood loss was measured by the weight of the swabs (postoperative swab weight subtracted by the preoperative weight) and the blood collected during the procedure.

\section{Screening criteria}

Adverse outcomes were collected for analysis, which included estimated blood loss ( $\geq 500 \mathrm{ml}$ ), conversion to CLS (an additional 5-mm trocar was inserted in the right or left lower quadrant in the LESS group), and complications. Complications were defined based on the Clavien-Dindo classification, which was grade II-V that occurred during the surgery, or any readmission and reoperation that occurred within 30 days after the first operation [11]. The classifications of surgical complications were as follows: (1) grade I, any deviation from the normal postoperative course without the need for pharmacological treatment or surgical, endoscopic, and radiological interventions; (2) grade II: requiring pharmacological treatment with drugs other than those allowed for grade I complications, including blood transfusions and total parenteral nutrition; (3) grade III: requiring surgical, endoscopic, or radiological intervention; grade III-a: intervention not under general anesthesia; grade III-b: intervention under general anesthesia; (4) grade IV: life-threatening complications (including CNS complications), requiring IC/ ICU-management; grade IV-a: single organ dysfunction (including dialysis); Grade IV-b: multi-organ dysfunction; and (5) grade V: the death of a patient.

The following conditions were determined as an adverse outcome of LESS, thus disqualifying patients to undergo LESS: 1) patients who had surgical complications, which were described as Clavien-Dindo classification II-V, readmission, and reoperation; 2) patients for whom the surgical method was converted from LESS to CLS or laparotomy during the operative procedure; 3) patients who underwent LESS with an estimated blood loss of $>500 \mathrm{ml}$; 4) patients who underwent CLS and had surgical complications, which were described as Clavien-Dindo classification II-V, readmission, and reoperation.

\section{Modeling procedures}

The model was built in Python using a Scikitlearn machine learning framework. Then the data were randomly split into training and validation sets with a $7: 3$ ratio. The training set data was used to build the model, and 10-fold cross validation was used to test the model and optimize the parameters. Last, the validation set data were used to verify the accuracy of the model. The clinical indicator data for LESS and CLS were collected later for model validation to improve the model accuracy. To further evaluate the models, receiver operating characteristic (ROC) curves were generated, and area under the curve (AUC) and precision-recall (PR) AUC were calculated. The AUC was used to compare different evaluation models.

\section{Results}

\section{Clinicopathological characteristics of patients and surgeons}

We finally identified 1019 cases of LESS and 1055 cases of CLS, which were grouped according to different surgical methods including adnexal surgery, myomectomy, hysterectomy, and tumor surgery. The clinicopathologic variables, including age, $\mathrm{BMI}$, and abdominal surgery history, were collected retrospectively from the medical records in Table I. No significant differences in the characteristics and clinical data of patients between the two groups were found for age, BMI, lesion size, menopausal status, and fertility history $(p \geq 0.05)$. There were no significant differences between the training and validated group of LESS and CLS. There were also no significant differences between the two groups for benign and malignant gynecological diseases $(p \geq$ 0.05). Both groups were comparable regarding their clinical characteristics. Figure 1 shows the correlation between different indicators and the correlation between indicators and labels. A total of $58.33 \%$ $(7 / 12)$ of the surgeons had surgery experience of $>500$ cases in LESS and 60\% (9/15) had surgery experience of $>500$ cases in CLS.

\section{Surgical outcomes of the patients}

The surgical-related data of the four different procedures are listed in Table II. We found significant differences in operative time, estimated blood loss, and $\delta \mathrm{Hb}$ between LESS and CLS for myomectomy ( $p=0.003, p=0.015, p=0.013$, respectively) and tumor surgery $(p=0.019, p=0.046, p=0.047$, respectively). The hospital stay of the patients after 
Table I. Clinical characteristics of 2074 included patients

\begin{tabular}{|c|c|c|c|c|c|c|c|}
\hline \multirow[t]{2}{*}{ Features } & \multicolumn{3}{|c|}{ LESS $(n=1019)$} & \multicolumn{3}{|c|}{$\operatorname{CLS}(n=1055)$} & \multirow{2}{*}{$\begin{array}{c}P \text {-value } \\
\text { (LESS } \\
\text { and } \\
\text { CLS) }\end{array}$} \\
\hline & $\begin{array}{l}\text { Training } \\
\text { group } \\
(n=713)\end{array}$ & $\begin{array}{l}\text { Validated } \\
\text { group } \\
(n=306)\end{array}$ & $P$-value & $\begin{array}{l}\text { Training group } \\
\quad(n=739)\end{array}$ & $\begin{array}{l}\text { Validated } \\
\text { group } \\
(n=316)\end{array}$ & $P$-value & \\
\hline Age [years] & $41.97 \pm 8.46$ & $42.31 \pm 7.68$ & 0.546 & $45.01 \pm 9.54$ & $44.18 \pm 8.95$ & 0.188 & 0.116 \\
\hline $\mathrm{BMI}\left[\mathrm{kg} / \mathrm{m}^{2}\right]$ & $23.64 \pm 2.44$ & $23.41 \pm 2.15$ & 0.154 & $23.23 \pm 2.45$ & $22.97 \pm 2.89$ & 0.136 & 0.146 \\
\hline Lesion size [mm] & $51.94 \pm 6.14$ & $52.42 \pm 7.25$ & 0.280 & $57.98 \pm 5.82$ & $58.54 \pm 4.68$ & 0.130 & 0.066 \\
\hline Menopausal status, $n(\%)$ : & & & 0.569 & & & 0.631 & 0.444 \\
\hline Pre/peri-menopause & $593(28.59 \%)$ & $250(12.05 \%)$ & - & $618(29.80 \%)$ & $268(12.92 \%)$ & - & - \\
\hline Post-menopause & $120(5.79 \%)$ & $56(2.70 \%)$ & - & $121(5.83 \%)$ & $48(2.31 \%)$ & - & - \\
\hline Fertility history, $n(\%)$ : & & & 0.595 & & & 0.652 & 0.409 \\
\hline $0-1$ & $584(28.16 \%)$ & $255(12.30 \%)$ & - & $621(29.94 \%)$ & $262(12.63 \%)$ & - & - \\
\hline$\geq 2$ & $129(6.22 \%)$ & $51(2.46 \%)$ & - & $118(5.69 \%)$ & $54(2.60 \%)$ & - & - \\
\hline \multicolumn{2}{|c|}{ Abdominal surgery history, $n(\%)$ : } & & 0.944 & & & 0.375 & 0.121 \\
\hline With abdominal surgery & $223(10.75 \%)$ & $95(4.58 \%)$ & - & $248(11.96 \%)$ & $115(5.54 \%)$ & - & - \\
\hline No abdominal surgery & $490(23.63 \%)$ & $211(10.17 \%)$ & - & $491(23.67 \%)$ & $201(9.69 \%)$ & - & - \\
\hline \multicolumn{4}{|c|}{ Benign gynecological diseases $(n=1918)$ : } & & & & 0.451 \\
\hline Adnexal surgery, $n(\%)$ & $424(20.44 \%)$ & $180(8.68 \%)$ & - & 415 (20.01\%) & $176(8.49 \%)$ & - & - \\
\hline Myomectomy, $n(\%)$ & $85(4.10 \%)$ & $40(1.93 \%)$ & - & $104(5.01 \%)$ & $41(1.98 \%)$ & - & - \\
\hline Hysterectomy, $n$ (\%) & $159(7.67 \%)$ & $66(3.18 \%)$ & - & $158(7.62 \%)$ & $70(3.38 \%)$ & - & - \\
\hline \multicolumn{4}{|c|}{ Malignant gynecological diseases $(n=156)$ : } & & & & 0.353 \\
\hline Endometrial cancer, $n(\%)$ & $9(0.43 \%)$ & $5(0.24 \%)$ & - & $20(0.96 \%)$ & $9(0.43 \%)$ & - & - \\
\hline Cervical cancer, $n(\%)$ & $30(1.45 \%)$ & $12(0.58 \%)$ & - & $34(1.64 \%)$ & $16(0.77 \%)$ & - & - \\
\hline Ovarian cancer, $n(\%)$ & $6(0.29 \%)$ & $3(0.14 \%)$ & - & $8(0.39 \%)$ & $4(0.19 \%)$ & - & - \\
\hline
\end{tabular}

surgery in the LESS group who underwent adnexal surgery, myomectomy, and hysterectomy were significantly shorter than the CLS group. The VAS $12 \mathrm{~h}$ or $24 \mathrm{~h}$ and VSS of the patients in the LESS group were also significantly lower than those in the CLS group. All cases were successfully performed without conversion to laparotomy. However, an additional 5-mm trocar was inserted in the right or left lower quadrant in the LESS group to allow the use of a third instrument for additional tissue retraction or manipulation. There were 46 (7.6\%), 14 (11.2\%), 12 (5.3\%), and $8(12.3 \%)$ cases that converted to CLS for adnexal surgery, myomectomy, hysterectomy, and tumor surgery, respectively. A total of 80 (7.85\%) cases experienced a conversion to multiport surgery, which mainly occurred in patients with severe pelvic adhesions $(n=32)$, lesions located in the posterior wall of the uterus $(n=21)$, lesion size of $\geq 10 \mathrm{~cm}$ $(n=12)$, and intraoperative bleeding $(n=15)$. These were factors that made the operation more difficult. The umbilical incision healed well in all the patients. No incisional complications, including incisional hernias or wound complications, were encountered in either group. The complications of the LESS group were observed in 10 cases including intestinal injury $(n=1)$, bladder injury $(n=1)$, ureteral injury $(n=2)$, incomplete vaginal cuff healing $(n=1)$, neurovascular injury $(n=1)$, pelvic lymphocyst formation $(n=2)$, and urinary retention $(n=2)$; while the CLS group had 7 cases. There were no perioperative deaths in this study. There were 244 patients unqualified to undergo LESS. 


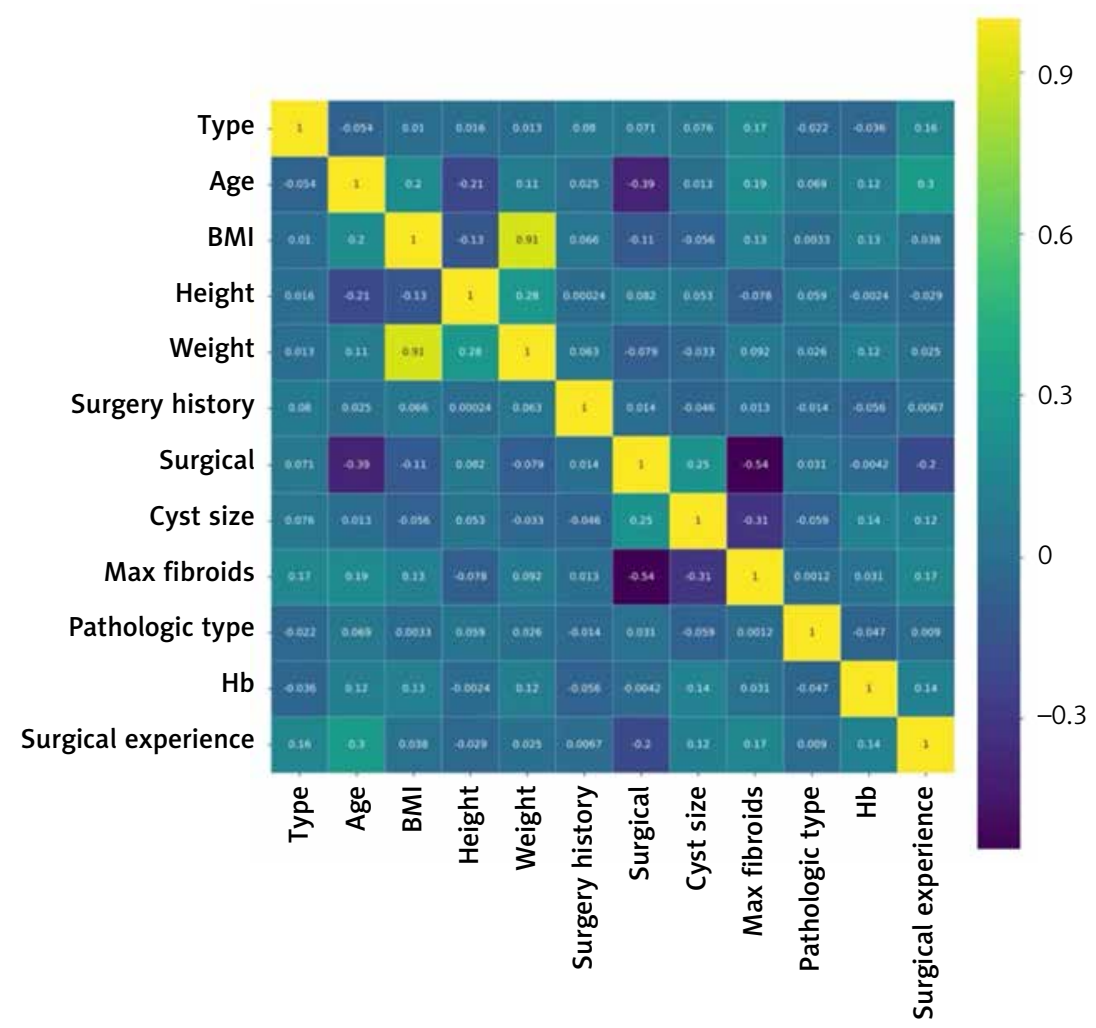

Figure 1. Thermal map of indicator correlation in the study. The closer the indicator is to yellow, the higher the correlation of the indicators. Pearson correlation of features

\section{Predicting adverse operative outcomes of LESS in gynecology using machine learning}

The data of patients were randomly divided into training and validation sets with a $7: 3$ ratio. Four algorithms (GBDT, XGBoost, random forest, and logistic regression) were used to build models for the previously ranked indicators. Table III shows the weight indicators for each algorithm. The classification models were designed. The receiver operating characteristic (ROC) curves of the four models are presented in Figure 2. The performance data of each model are presented in Table IV. The GBDT model achieved the highest accuracy at 0.8 , followed by the XGBoost model (0.795). The XGBoost and random forest model had both the largest AUC of 0.77, with an AUC PR of 0.64 and 0.63. The second-largest AUC was GBDT (0.76). The clinical weight indicators of each algorithm are shown in Table III. The maximum fibroid size was the most important predictor in GBDT and random forest. These findings are concordant with the successful completion of LESS. The decision tree model had a good operative outcome compared with the linear model.

\section{Discussion}

We aimed to compare the clinical outcomes of LESS and conventional CLS in gynecological diseases and to construct a LESS risk prediction model based on multiple machine learning algorithms. In our study LESS showed some advantages in postoperative outcomes compared with CLS. We identified the decision tree model as the superior prediction model for LESS.

Surgeons must maintain and strive to further improve the minimally invasive advantages of laparoscopy. Simultaneously, they must continuously endeavor to expand the surgical indications of laparoscopy to include more complicated and difficult surgical procedures. This is the driving force behind the development of laparoscopic surgical methods.

An advantage of our study is the inclusion of a large population of subjects with 2074 cases of benign and malignant gynecological diseases treated with LESS and CLS who were enrolled from a single center. During the study period, there was no fundamental difference in lesion size or abdominal surgery history between patients who underwent LESS 


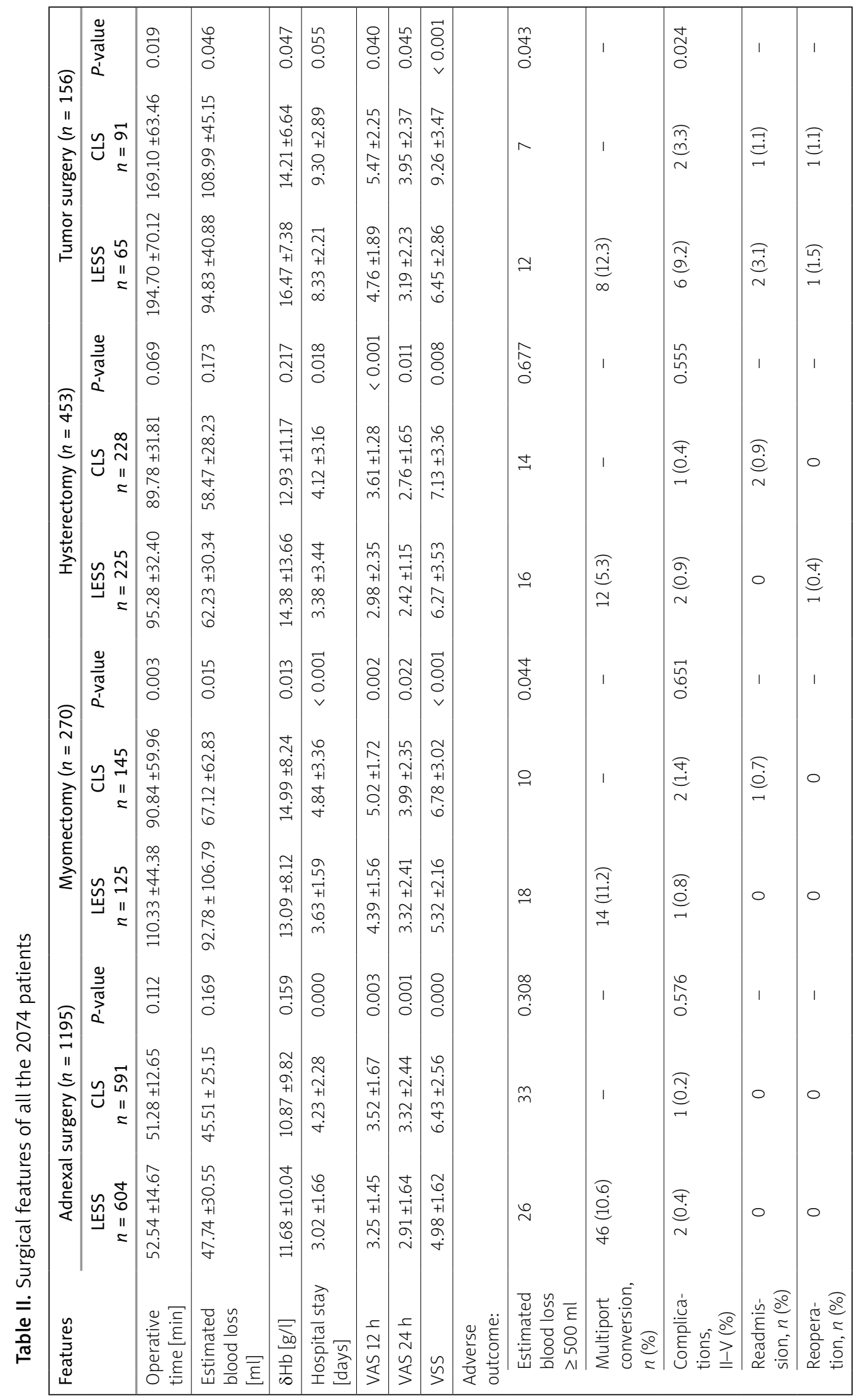


Table III. Summary of clinical weight indicators in each algorithm

\begin{tabular}{|lcccc|}
\hline Features & GBDT & XGBoost & Random forest & Logistic regression \\
\hline Maximum fibroids & 0.223797229 & 0.113070790 & 0.195771614 & -0.000024900 \\
\hline Surgical & 0.185641380 & 0.067407586 & 0.144294121 & 0.177613252 \\
\hline Cyst size & 0.160775968 & 0.096883304 & 0.144869930 & 0.020854078 \\
\hline Surgical experience & 0.105421413 & 0.077796570 & 0.117731356 & 0.502856825 \\
\hline $\mathrm{Hb}$ & 0.095751750 & 0.182652820 & 0.094800881 & -0.010101314 \\
\hline BMl & 0.083467713 & 0.153901900 & 0.083226945 & 0.026878383 \\
\hline Age & 0.078454350 & 0.119110900 & 0.099469879 & -0.016817502 \\
\hline Weight & 0.038330223 & 0.073206090 & 0.045950095 & 0.002249880 \\
\hline Surgical history & 0.011549793 & 0.020053154 & 0.011218476 & 0.359279089 \\
\hline Height & 0.010430133 & 0.090359990 & 0.050124431 & -0.013225431 \\
\hline Pathologic type & 0.006380049 & 0.005556898 & 0.012542272 & -0.006831201 \\
\hline
\end{tabular}

Eleven clinical indicators were ranked according to the weight ratios in each of the four algorithms. The weight ratios of each algorithm were different.

A

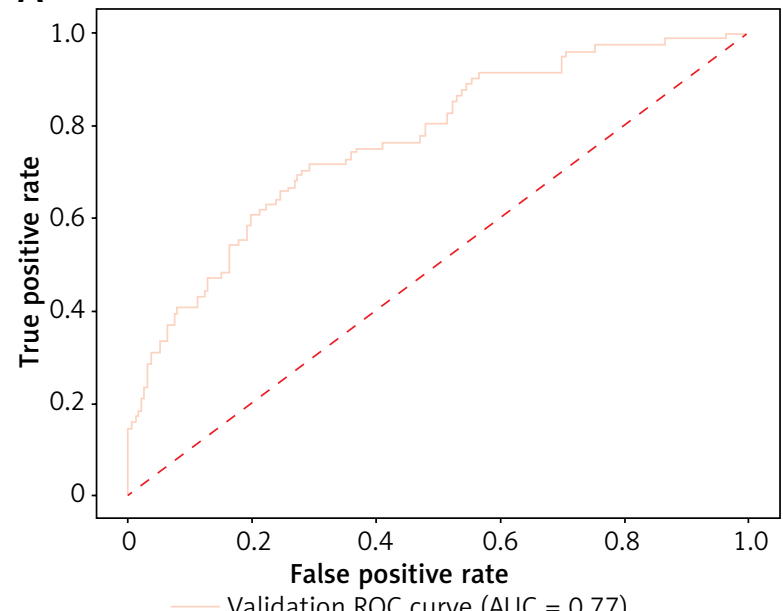

$c$

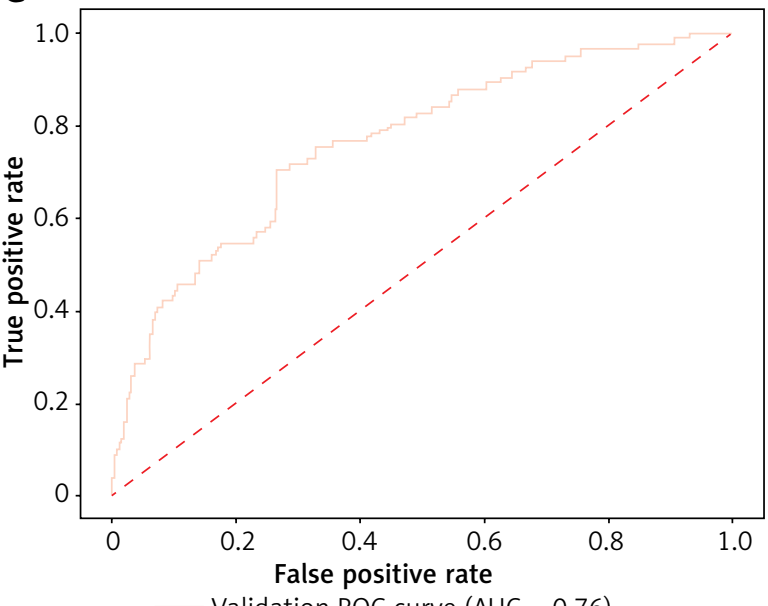

B

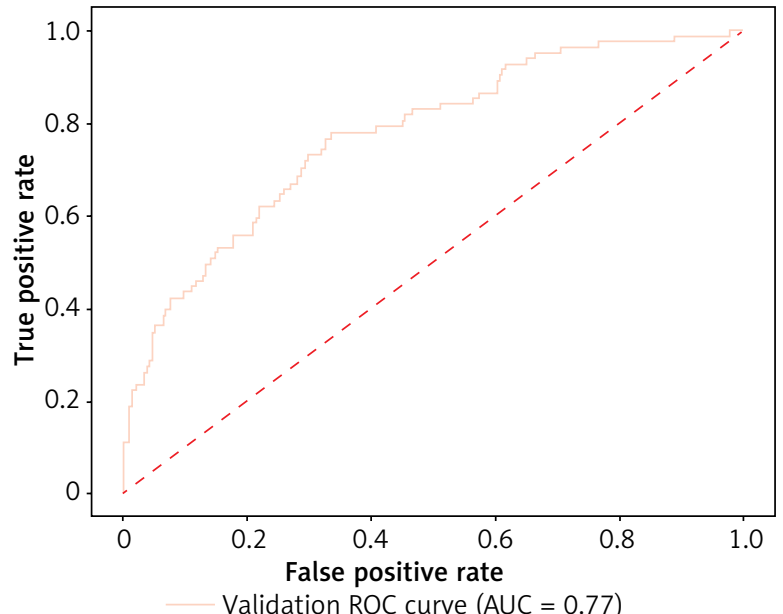

D

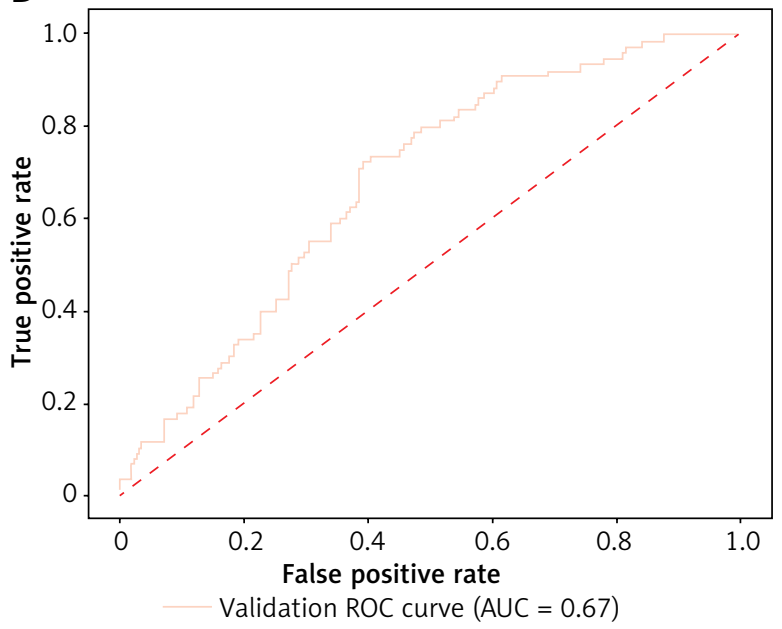

Figure 2. Receiver operating characteristic (ROC) curve of (A) XGBoost model, (B) random forest model, (C) GBDT model, and (D) logistic regression model 
Table IV. Machine learning model performance

\begin{tabular}{|lcccc|}
\hline Factor & GBDT & XGBoost & Random forest & Logistic regression \\
\hline Accuracy & 0.80 & 0.795 & 0.79 & 0.75 \\
\hline Cut-off & 0.22 & 0.22 & 0.22 & 0.23 \\
\hline Sensitivity & 0.68 & 0.70 & 0.70 & 0.72 \\
\hline Specificity & 0.69 & 0.72 & 0.71 & 0.60 \\
\hline AUC-Validation ROC & 0.76 & 0.77 & 0.77 & 0.67 \\
\hline AUC-PR & 0.60 & 0.64 & 0.63 & 0.49 \\
\hline
\end{tabular}

and CLS. The other advantages of LESS over the CLS approach include improved cosmesis, increased patient satisfaction, decreased postoperative pain, shorter hospital stay, and decreased risk of traumatic tissue or vascular injury with trocar placement [12-14]. Although CLS using several trocars may be less invasive than laparotomy, patients undergoing this type of CLS acquire multiple postoperative scars on the abdominal wall. In LESS, the surgical procedure is performed using a single incision on the umbilicus. Because the incision site can be concealed in the umbilicus, the postoperative wound is minimized and cosmetically improved.

Many authors have compared the reliability of the observer portion of the assessment tool using the VSS and found it to be superior to the most widely accepted scale [15]. The results of a current meta-analysis showed a benefit of LESS over CLS in terms of cosmetic patient satisfaction due to the smaller number of trocars inserted into the abdomen [16]. Our results also showed that there was a significant difference in the VSS scores between the LESS and CLS groups in the cosmetic outcome. Having only one postoperative scar on the patient's abdominal wall increased patient satisfaction. LESS has fewer ports than CLS. Postoperative pain is also perceived to be less. Although the pain felt by individual patients is very subjective, CLS is still reported to be more painful than LESS [17]. In our study, the LESS group showed improved postoperative pain in the $12 / 24 \mathrm{~h}$ VAS, which demonstrated that fewer scars could translate to reduced perceived pain.

Due to the lack of triangulation, myomectomy and tumor surgery in LESS, which is more difficult to perform and requires plenty of suturing and separating in special angles, showed longer operative times and more blood loss. In benign gynecological diseases, we noted a shorter postoperative hospital stay in the LESS group. Better outcomes were achieved by
LESS in terms of cosmesis, postoperative pain, and hospital stay in patients with benign gynecological diseases. In the CLS group, better outcomes were noted for tumor procedures with fewer complications compared to LESS. Therefore, surgeons must choose the appropriate surgical method for better postoperative outcomes.

When we discussed the surgical method preoperatively, decisions on the method of choice were made based on medical history, physical examination, and imaging findings. Not every patient is suitable for LESS. We believe that an inappropriate surgical method will cause a longer operative time, increase in intraoperative bleeding, higher surgeon fatigue, increase in the rate of conversion to multisite surgery, increase in the number of complications, and other adverse effects. We embrace the concept that patient safety comes first. The use of an additional trocar could facilitate the procedure, reduce the risk of complications, and allow for precise surgical resection. Notably, the use of one additional trocar was applied in $7.85 \%$ of the LESS procedures performed in our study. A previous model was used to analyze the risks of conversion and postoperative complications in urological laparoendoscopic single-site surgery. The factors significantly associated with the risk of conversion were oncological surgical indication, pelvic surgery, robotic approach, extended operative time and an intraoperative complication. The conversion rate suggested that early adopters of the technique had adhered to the principles of careful patient selection and safety [18]. It is necessary to find an evaluation method that can be used to support preoperative clinical decision-making and referencing. Through proper preoperative evaluation based on the characteristics of a patient, we will be able to predict the risk of adverse surgical conditions. In this way, surgeons will have more information when selecting the surgical method. 
Machine learning models have achieved rapid development and are widely used in learning modeling and medical case prediction [19-21]. The systems are made up of three major parts, which are: (1) model: the system that makes predictions or identifications; (2) parameters: the signals or factors used by the model to form its decisions; (3) learner: the system that adjusts the parameters - and in turn the model by looking at differences in predictions versus actual outcome. The advantage of the decision tree model includes carrying out visual analysis, extracting rules easily, processing nominal and numerical data at the same time, and dealing with irrelevant features. The linear model on the other hand is suitable for dealing with a linear relationship. Our study was based on practical clinical issues and established an evaluation and prediction model, and ultimately, an optimal model was selected to analyze key clinical factors to predict the occurrence of adverse surgical conditions. The risk prediction model is based on machine learning algorithms which can predict the risk of adverse surgical conditions. It facilitated clinical decision-making and referencing before and during an operation. At present, there is no existing clinical prediction model used for preoperative evaluation of LESS. So far, there is also no similar work for the prediction model with machine learning classification of LESS.

Our results showed that among the LESS prediction models, the AUC values of the validation sets for the four prediction models were both $\geq 0.65$, indicating a good predictive value for LESS. The AUC value of the XGBoost and random forest algorithm was 0.77 for the validation sets and the GBDT came next, which were better than the AUC values of the logistic regression. Therefore, the decision tree model is more suitable for the prediction of LESS for gynecologic diseases. With this model, fibroid size, surgical method, cyst size, surgical experience, and $\mathrm{Hb}$ concentration ranked highly in weight ratios for LESS gynecologic procedures. These factors were associated with the successful completion of LESS. This conforms to the actual conditions of the LESS. Fibroid and cyst sizes were shown to be pre-conditions for successful LESS performance. Different intra-operative circumstances may require a change in the surgical method. These issues are also fundamental factors that result in intraoperative bleeding. All of the above findings are specific criteria for the successful implementation of LESS. The surgical experience did not have a significant influence on the change in surgical method, which might be because LESS for benign gynecologic disease was less difficult than tumor procedures. The surgical experience did not rank highly among the most influential factors. For example, a surgeon with fewer cases of laparoscopic surgery seldom performs complicated operations such as uterine surgery. The preoperative $\mathrm{Hb}$ concentration was also included because it could lead to changes in the surgical method. If the patient was anemic or bleeding occurred during the operation, the surgical method could be converted during the operation. Therefore, if the patient's anemia is corrected preoperatively, the possibility of successful completion of LESS would increase.

While preparing one gynecology surgery, each surgeon can input the clinical indicators of the patient, and then get the value risk of adverse outcomes by the machine learning model. Then the surgeon can choose the proper operation method, which can minimize the adverse outcomes such as bleeding and complications. During the operative process, whether or not LESS should be converted to multi-site surgery is not only based on the surgeon's clinical judgement but also on the machine learning outcomes as well. The decision depends on the complexity of the surgery, as well as the patient's body size, fibroid size, ovarian cyst size, preoperative $\mathrm{Hb}$ concentration, and surgical procedure. It provides valid information for a surgeon preoperatively, which can be used as a reference to support clinical decision-making. It can also help the surgeon to take better control of surgical indications. If the risk of adverse conditions may be high, the surgeon should convert to CLS during LESS as soon as possible to reduce and avoid severe complications.

This study had some limitations. This was a retrospective study conducted in a single center, which may limit the external validity of the analysis and generalizability of the study, as well as introducing inherent biases. Another limitation is observation bias based on the subjective experience of the selected surgeons. Moreover, these data reflected results from different experienced surgeons in laparoscopy. In the future, large-scale randomized and double-blinded prospective trials should be performed to validate the use of LESS in gynecological surgery in terms of predicting patient outcomes. We will further conduct multi-center studies, expand the sample size, enhance the value of prediction, improve the model accuracy, and enrich the models that may cover more gynecologic tumor types. 
With the number of cases being more adequate, we can precisely predict and evaluate the model according to the personal data of surgeons, analyze which factors influence the final clinical outcome, select and include the factors used to build a model, and analyze the weight of each factor to generate individualized data for every surgeon. This allows a more objective clinical decision during preoperative evaluation based on a large data set. Thus, the most suitable surgical method can be selected to achieve optimal clinical therapeutic effects. The clinical application of LESS will be more evidence-based and patient-oriented, using machine learning to evaluate each patient's circumstances, surgeon's abilities, and evaluate outcomes, and create an algorithm that calculates the more predictive variables that can lead to the desired outcomes of shorter hospital stay, less pain, least recurrence, and least blood loss. There will be more optimal and accurate prediction models that provide a more reliable basis with sufficient evidence-based reference for the evolution of clinical guidelines and medical decisions.

\section{Conclusions}

LESS is more favorable than CLS in reducing postoperative pain, enhancing postoperative recovery, shortening hospital stay, and providing better esthetic results. The risk prediction model based on a machine learning algorithm has manifested a high degree of accuracy and can satisfy the requirements of the doctors' demand for individualized preoperative evaluation and surgical safety in LESS.

\section{Acknowledgments}

Jun Ma and Jiani Yang contributed equally to the article.

\section{Conflict of interest}

The authors declare no conflict of interest.

\section{References}

1. Chapron C, Querleu D, Bruhat MA, et al. Surgical complications of diagnostic and operative gynaecological laparoscopy: a series of 29,966 cases. Hum Reprod 1998; 13: 867-72.

2. Miotto R, Li L, Kidd BA, Dudley JT. Deep patient: an unsupervised representation to predict the future of patients from the electronic health records. Sci Rep 2016; 6: 26094.

3. Han L, Yuan Y, Zheng S, et al. The pan-cancer analysis of pseudogene expression reveals biologically and clinically relevant tumour subtypes. Nat Commun 2014; 5: 3963.
4. Friedman J. Greedy function approximation: a gradient boosting machine. Ann Statistics 2001; 29: 1189-232.

5. Xuan P, Sun C, Zhang T, et al. Gradient boosting decision treebased method for predicting interactions between target genes and drugs. Front Genet 2019; 10: 459.

6. Xie Y, Jiang B, Gong E, et al. Use of gradient boosting machine learning to predict patient outcome in acute ischemic stroke on the basis of imaging, demographic, and clinical information. AJR Am J Roentgenol 2019; 212: 44-51.

7. Zhang C, Zheng Y, Ma X, Han J. Assembler: efficient discovery of spatial co-evolving patterns in massive geo-sensory data. KDD 2015; 2015: 1415-24.

8. Strobl C, Boulesteix AL, Kneib T, et al. Conditional variable importance for random forests. BMC Bioinformatics 2008; 9: 307.

9. Paik ES, Choi HJ, Kim TJ, et al. Molecular signature for lymphatic invasion associated with survival of epithelial ovarian cancer. Cancer Res Treat 2018; 50: 461-73.

10. Wal MB, Verhaegen PD, Middelkoop E, Zuijlen PP. A clinimetric overview of scar assessment scales. J Burn Care Res 2012; 33 : e79-87.

11. Clavien PA, Barkun J, Oliveira ML, et al. The Clavien-Dindo classification of surgical complications: five-year experience. Ann Surg 2009; 250: 187-96.

12. Yim GW, Jung YW, Paek J, et al. Transumbilical single-port access versus conventional total laparoscopic hysterectomy: surgical outcomes. Am J Obstet Gynecol 2010; 203: 26 e1-6.

13. Jung YW, Kim YT, Lee DW, et al. The feasibility of scarless single-port transumbilical total laparoscopic hysterectomy: initial clinical experience. Surg Endosc 2010; 24: 1686-92.

14. Ghezzi F, Cromi A, Colombo G, et al. Minimizing ancillary ports size in gynecologic laparoscopy: a randomized trial. J Minim Invasive Gynecol 2005; 12: 480-5.

15. Draaijers LJ, Tempelman FR, Botman YA, et al. The patient and observer scar assessment scale: a reliable and feasible tool for scar evaluation. Plast Reconstr Surg 2004; 113: 1960-5.

16. Haueter R, Schutz T, Raptis DA, et al. Meta-analysis of singleport versus conventional laparoscopic cholecystectomy comparing body image and cosmesis. Br J Surg 2017; 104: 1141-59.

17. Park JY, Kim DY, Suh DS, et al. Laparoendoscopic single-site versus conventional laparoscopic surgery for ovarian mature cystic teratoma. Obstet Gynecol Sci 2015; 58: 294-301.

18. Autorino R, Kaouk JH, Yakoubi R, et al. Urological laparoendoscopic single site surgery: multi-institutional analysis of risk factors for conversion and postoperative complications. J Urol 2012; 187: 1989-94.

19. Stehrer R, Hingsammer L, Staudigl C, et al. Machine learning based prediction of perioperative blood loss in orthognathic surgery. J Craniomaxillofac Surg 2019; 47: 1676-81.

20. Lundberg SM, Nair B, Vavilala MS, et al. Explainable machinelearning predictions for the prevention of hypoxaemia during surgery. Nat Biomed Eng 2018; 2: 749-60.

21. Chu CS, Lee NP, Adeoye J, et al. Machine learning and treatment outcome prediction for oral cancer. J Oral Pathol Med 2020; 49: 977-85.

Received: 9.03.2021, accepted: 6.04.2021. 